\title{
Fully automated 5-plex fluorescent immunohistochemistry with tyramide signal amplification and same species antibodies
}

\author{
Wenjun Zhang ${ }^{1,2}$, Antony Hubbard ${ }^{1,2}$, Tobin Jones ${ }^{1}$, Adriana Racolta' ${ }^{1}$, Srabani Bhaumik ${ }^{1}$, Nick Cummins ${ }^{1}$, \\ Liping Zhang ${ }^{1}$, Karl Garsha', Frank Ventura ${ }^{1}$, Mark R Lefever ${ }^{1}$, Zhenqiang Lu', John K Hurley ${ }^{1}$, William A Day ${ }^{1}$, \\ Lidija Pestic-Dragovich', Larry E Morrison ${ }^{1}$ and Lei Tang ${ }^{1}$
}

The ability to simultaneously visualize the presence, abundance, location and functional state of many targets in cells and tissues has been described as a true next-generation approach in immunohistochemistry (IHC). A typical requirement for multiplex IHC ( $\mathrm{mIHC})$ is the use of different animal species for each primary $\left(1^{\circ} \mathrm{Ab}\right)$ and secondary $\left(2^{\circ} \mathrm{Ab}\right)$ antibody pair. Although $1^{\circ} \mathrm{Abs}$ from different species have been used with differently labeled species-specific $2^{\circ} \mathrm{Abs}$, quite often the appropriate combination of antibodies is not available. More recently, sequential detection of multiple antigens using $1^{\circ} \mathrm{Abs}$ from the same species used a microwaving treatment between successive antigen detection cycles to elute previously bound $1^{\circ} \mathrm{Ab} / 2^{\circ} \mathrm{Ab}$ complex and therefore to prevent the cross-reactivity of anti-species $2^{\circ} \mathrm{Abs}$ used in subsequent detection cycles. We present here a fully automated $1^{\circ} \mathrm{Ab} / 2^{\circ} \mathrm{Ab}$ complex heat deactivation (HD) method on Ventana's BenchMark ULTRA slide stainer. This method is applied to detection using fluorophore-conjugated tyramide deposited on the tissue and takes advantage of the strong covalent bonding of the detection substrate to the tissue, preventing its elution in the HD process. The HD process was characterized for (1) effectiveness in preventing Ab crossreactivity, (2) impact on the epitopes and (3) impact on the fluorophores. An automated 5-plex fluorescent IHC assay was further developed using the HD method and rabbit $1^{\circ} \mathrm{Abs}$ for CD3, CD8, CD20, CD68 and FoxP3 immune biomarkers in human tissue specimens. The fluorophores were carefully chosen and the narrow-band filters were designed to allow visualization of the staining under fluorescent microscope with minimal bleed through. The automated 5-plex fluorescent IHC assay achieved staining results comparable to the respective single-plex chromogenic IHC assays. This technology enables automated $\mathrm{mlHC}$ using unmodified $1^{\circ} \mathrm{Abs}$ from same species and the corresponding anti-species $2^{\circ} \mathrm{Ab}$ on a clinically established automated platform to ensure staining quality, reliability and reproducibility.

Laboratory Investigation (2017) 97, 873-885; doi:10.1038/labinvest.2017.37; published online 15 May 2017

Immunohistochemical (IHC) detection of multiple antigens on the same tissue section (multiplex IHC (mIHC) $)^{1-8}$ represents a major unmet technological need in research and clinical diagnostics. ${ }^{9}$ The simplest IHC approach is the use of direct detection of antigens by (same or different species) primary antibodies $\left(1^{\circ} \mathrm{Ab}\right)$ that are directly labeled with different fluorophores. ${ }^{10-13}$ Some disadvantages of using direct detection are (a) lower sensitivity, compared with amplified detection, (b) a need for sizeable quantities of conjugated antibodies, usually also more expensive and (c) risk that antibody activity may be adversely affected by direct labeling. The indirect IHC detection method uses two or more layers of antibodies, allowing for amplification of signal and increased sensitivity. For example, using available $1^{\circ} \mathrm{Abs}$ from different species for double or multiple antigen detection is possible when used in combination with differently labeled species-specific secondary antibodies $\left(2^{\circ} \mathrm{Abs}\right)$ in mIHC. ${ }^{3,10,14,15}$ However, quite often the appropriate combination of different species antibodies is not available and same species antibodies are used while using a method for their inactivation between successive cycles of immunodetection.

\footnotetext{
VVentana Medical Systems, Tucson, AZ, USA

Correspondence: Dr L Tang, PhD, Ventana Medical Systems, 1910 E Innovation Park Drive, Tucson, AZ 85755, USA.

E-mail: lei.tang@roche.com

${ }^{2}$ These authors contributed equally to this work.

Received 22 December 2016; revised 24 February 2017; accepted 25 February 2017
} 
One approach to prevent cross-reactivity during sequential multiplex detection is the microwaving treatment between staining cycles preventing the anti-species $2^{\circ} \mathrm{Ab}$ in subsequent staining cycles from binding to the antigen-bound $1^{\circ} \mathrm{Ab}$ used in the previous staining cycles. ${ }^{4-8,15-18}$ Extensive evaluation of microwaving effectiveness on abolishing cross-reactive staining signals and its effect on loss of fluorescence for select fluorophores $^{6,7}$ is yet to be performed. In addition, microwaving is prone to remove some delicate biopsies from polylysine slides, especially if they have already been antigen retrieved by a previous heat-mediated procedure. ${ }^{19}$

Another approach for preventing cross-reactivity in $\mathrm{mIHC}$ detection is antibody elution. ${ }^{19,20}$ A number of buffers with different $\mathrm{pH}$, osmolarity, detergent content and denaturing properties were tested to strip the already bound antibody complex from previous IHC staining cycles. Glycine-HCl/ sodium dodecyl sulfate (SDS) $\mathrm{pH} 2$ or 2-mercaptoethanol/SDS pH 6.75 was reported to be effective by one group but not the other. In addition, certain buffers were found to be hazardous, decolorized $\mathrm{H} \& \mathrm{E}$ stain and/or reduced nuclear protein target staining. We present here a fully automated mIHC technology using a thermochemical process to deactivate antibody complex between staining cycles on a commercial automated slide stainer. This technology allows the use of $1^{\circ} \mathrm{Abs}$ from the same species, detected by the antispecies $2^{\circ} \mathrm{Abs}$ conjugated to HRP, which in turn drives in-situ deposition of tyramide-fluorophores (Ty-fluor). We demonstrated the technology feasibility by detecting CD20, CD3, CD8, CD68 and FoxP3 on a single slide. Our carefully selected five fluorophores and narrow-band filter designs reduce bleed through of neighboring fluorophores and enable visiualization of the signals under the fluorescence microscope. In addition, Ty-fluors yield enhanced sensitivity.

\section{MATERIALS AND METHODS}

\section{Specimens}

Individual tissue samples obtained from an archive maintained at Ventana Medical Systems (Tucson, AZ, USA) were used in this study. These samples were redundant clinical specimens that had been de-identified and unlinked from patient information. Tumor tissue microarray slides were obtained from Pantomics (Richmond, CA, USA). Paraffin sections $(4 \mu \mathrm{m})$ containing tissue cores of formalin-fixed, paraffin-embedded breast tissue were mounted on Superfrost Plus glass slides.

\section{Automated IHC}

IHC assays were performed on a VENTANA BenchMark Ultra automated staining instrument (Ventana Medical Systems), using VENTANA reagents except as noted, according to the manufacturer's instructions. Slides were de-paraffinized using EZ Prep solution (cat \# 950-102) for $16 \mathrm{~min}$ at $72{ }^{\circ} \mathrm{C}$. Epitope retrieval was accomplished with CC1 solution (cat \# 950-224) at high temperature (eg, 95-100 ${ }^{\circ} \mathrm{C}$ ) for a period of time (eg, 32-92 min) that is suitable for a specific tissue type. Rabbit $1^{\circ}$ Abs (listed in Table 1) were titered with a TBS antibody diluent (part\# 90103) into user fillable dispensers for use on the automated stainer. For brightfield detection, slides were developed using the VENTANA ultraView Universal DAB detection kit (cat \# 760-500) according to the manufacturer's instructions. Slides were then counterstained with hematoxylin II (cat \# 7902208) for $8 \mathrm{~min}$, followed by Bluing reagent (cat \# 760-2037) for $4 \mathrm{~min}$. For fluorescent detection, the rabbit $1^{\circ} \mathrm{Abs}$ were incubated for $16 \mathrm{~min}$ with a goat anti-rabbit $2^{\circ} \mathrm{Ab}$ conjugated with horseradish peroxidase (GaR-HRP $2^{\circ} \mathrm{Ab}$ ) from VENTANA ultraView SISH DNP Detection Kit (cat \# 760-098). After two rinses with reaction buffer, a Ty-fluor is introduced and incubated for $4 \mathrm{~min}$, followed with the application of $0.01 \% \mathrm{H}_{2} \mathrm{O}_{2}$ (DISCOVERY reagent cat \# 760-244). The $\mathrm{HRP}$ in the $1^{\circ} \mathrm{Abs} / 2^{\circ} \mathrm{Ab}$ complex is then allowed to react with the Ty-fluor for $8 \mathrm{~min}$, which leads to oxidation and subsequent covalent binding of Ty-fluor to tyrosine residues in the vicinity of the antigens. Five Ty-fluor conjugates were prepared in-house as research reagents: ${ }^{21} 15 \mu \mathrm{M}$ carboxyrhodamine-6G-Tyramide (Ty-R6G, DISCOVERY reagent cat \# 760-244), $5 \mu \mathrm{M}$ sulforhodamine101-tyramide (Ty-Texas Red (TR)), $16 \mu \mathrm{M}$ diethylaminocoumarintyramide (Ty-DCC), $12 \mu \mathrm{M}$ SulphoCy5-Tyramide (Ty-Cy5) and $60 \mu \mathrm{M}$ fluorescein isothiocyanate -Tyramide (Ty-FITC). The slides were counterstained with QD DAPI (DISCOVERY reagent cat \# 760-4196), and coverslipped with ProLong Diamond Antifade Mountant (ThermoFisher Scientific, cat \# P36961).

Table 1 Primary antibodies ( $\left.1^{\circ} \mathrm{Abs}\right)$

\begin{tabular}{|c|c|c|c|c|c|c|}
\hline Name & Recommended dilution in $\mathrm{DAB}$ staining & Species & Clone & Location & Dilution in 5-plex FL & Source \\
\hline CD20 RUO & 1:100 & Rabbit & SP32 & Membrane & 1:140 & Spring Bioscience, cat \# M3324 \\
\hline FoxP3 RUO & 1:100 & Rabbit & SP97 & Nucleus & 1:50 & Spring Bioscience, cat \# M3974 \\
\hline CD68 RUO & 1:100 & Rabbit & SP251 & Cytoplasm & $1: 20$ & Spring Bioscience, cat \# M5514 \\
\hline CD3 RUO & 1:150 & Rabbit & SP162 & Membrane & $1: 300$ & Spring Bioscience, cat \# M4624 \\
\hline CD8 RUO & 1:100 & Rabbit & SP239 & Membrane & 1:100 & Spring Bioscience, cat \# M5394 \\
\hline
\end{tabular}




\section{Imaging Acquisition and Analysis}

Instrumentation for both direct inspection of fluorescent slides and analytical fluorescence measurement of stained slides was integrated from commercially available components. Imaging was performed on an upright Zeiss AxioImager.M2 equipped with an encoded motorized stage, motorized $\mathrm{z}$ drive, filter turret, tube lens turret and objective turret (Zeiss, Thornwood, NY, USA). A $1 \times$ c-mount was used to capture images using a Photometrics CoolSNAP ES2 12-bit monochrome CCD camera (Photometrics, Tucson, AZ, USA) with closed-loop cooling to 0 degrees. Image capture was performed using Photometrics' 'alt-normal' readout clocking to extend quantum efficiency in the red part of the spectrum and $1 \times$ binning was used. Images were collected with a Plan-Apochromat 20x/0.8NA M27 objective lens (Zeiss) and an Exfo Exacte light source (Lumen Dynamics, Mississigua, Ontario) capable of repeatable illumination at the sample plane with $<1 \%$ variation in absolute illumination level.

The fluorescent filters were carefully selected/designed to minimize 'bleed through' from fluorophores with overlapping excitation and emission spectra. The fluoresce filters and cubes were obtained from Chroma Technology Corp. (Bellows Falls, VT, USA) and included the following filter sets: (1) ET DAPI cat \#49000 (AT350/50 excitation filter, ET460/50 emission filter, T400lp beamsplitter), (2) ET Aqua cat \#49302 (436/20 excitation filter, ET480/30 emission filter, T455lp beamsplitter), (3) custom Green (ET490/20 excitation filter, ET520/20 emission filter, T505lp beamsplitter), (4) ET Gold cat \# (ET546/11 excitation filter, ET572/23 emission filter, T556lpxr beamsplitter), (5) ET Red cat \#49306 (ET580/25 excitation filter, ET625/30 emission filter, T600lpxr beamsplitter), and (6) ET Cy5 Narrow Excitation cat \#49009 (ET640/30 excitation fitler, ET690/50 emission filter, T660lpxr beamsplitter).

Custom system automation and analysis software written in Python ('Ventana Analytical Imaging System' (VAIS)) was used to calibrate instrumentation, automate the data collection workflow and provide analysis of collected images for intensity distributions. ${ }^{22,23}$

For quantitative digital analysis, operators screened each slide under microscope and selected 10 representative regions of interest (ROI) for each stained biomarker and avoided areas containing highly autofluorescent red blood cells. These ROI were recorded in VAIS software and automated capture using standardized capture parameters for illumination and exposure was used to collect the data. Exposure times were standardized for each channel and chosen to nearly fill the dynamic range of the camera for each given marker. The exposure times ranged between 25 and $100 \mathrm{~ms}$. The stained structures in collected images were segmented from unstained (dark) areas using objective thresholding techniques. Where appropriate, the statistical-based Otsu method was used across images in a set to be compared. Alternatively, for situations where multiple distributions of intensity confounded the use of Otsu thresholds, an absolute threshold was set just above the empirically determined background level for a population of (10) images. The thresholds permitted measurement of only the pixels in stained structures so that staining intensity could be evaluated without dark background in images being included in the measurement. In order to compensate for biological differences in the area of stained structures image to image, a mean of the measured pixel values was taken (expressed as mean intensity thresholded pixels).

To subjectively evaluate staining localization and specificity in the reproducibility studies, trained readers visually assessed all of the slides in a given study for intensity and background at $\times 20$ magnification under the microscope. Subjective rating of specific staining intensity scales are: 0 , negative staining of the cells; $1+$, weak membrane staining of cells for CD3, CD8 and CD20, cytoplasmic staining for CD68, and nuclear staining for FoxP3; 2+, moderate membrane staining of cells for CD3, CD8 and CD20, cytoplasmic staining for CD68, and nuclear staining for FoxP3; 3+, strong membrane staining of cells for CD3, CD8 and CD20, cytoplasmic staining for CD68, and nuclear staining for FoxP3. 'Not evaluable' means that interpretation is not possible, for example, no tissue present, artifacts, etc. Scales for subjectively rating nonspecific background staining are: 0 , no background staining; 0.5 , some discernible nonspecific staining that is not obtrusive to interpretation of specific staining; 1, nonspecific staining is obtrusive to interpretation of specific staining; 2, nonspecific staining is extremely obtrusive but is not as intense as specific staining; 3, nonspecific staining cannot be differentiated from specific staining.

A Zeiss Axioscan.Z1 scanner (Jena, Germany) equipped with the same set of filters as outlined above was used for fluorescence whole-slide scanning. The instrument used in our study was retrofitted with a Lumen Dynamics Exacte calibrated light source as used on the upright microscopes in order to minimize fluctuation of intensities because of illumination drift. Zeiss Zen Slidescan and Zen Blue softwares were used to acquire and visualize images, respectively. The exposure time for individual channels was set to allow acquisition of fluorescent signals within the dynamic range of display setting.

In the study, comparing 5-plex fluorescent staining with single $\mathrm{DAB}$ staining, the images were visualized as outlined below. Tissue landmarks on whole-slide scans of both brightfield imaged DAB slides and fluorescent imaged slides were identified and centered in a split image viewer within the ZEN software suite, where individual fluorescent channel images from replicate slides in the reproducibility studies could be uploaded and viewed within the same window. The aligned and synchronized split images could be zoomed, moved or adjusted simultaneously to evaluate staining patterns, staining quality and percent coverage of each marker in the reproducibility studies. DAB image intensity scale was set between 0 and 225 with gamma $=1$. All five channels 
from the scanned fluorescent 5-plex stained slides were set between the intensity scale of 300-32 500, with gamma $=1$. A board-certified pathologist (co-author: L Zhang) evaluated the markers in fluorescence images with the counterparts in $\mathrm{DAB}$ images in the split image viewer. A subset of approximately $5-10 \%$ of the tissue area was reviewed at a higher magnification to confirm morphology and consistency across both fluorescence and DAB images. Channel visualization parameters of gamma and display intensity ramp were in some instances manually adjusted to minimize the impact of background staining and facilitate the evaluation.

\section{Automated Heat Deactivation (HD) Using CC2}

CC2 solution (Ventana cat \# 950-223) was used for HD. CC2 is a citrate/acetate-based buffer, $\mathrm{pH} 6.0$ and contains $0.3 \%$ SDS. After completion of a prior round of antigen detection, slides were rinsed with EZ Prep solution followed with application of $1.4 \mathrm{ml} \mathrm{CC2}$ solution. Heatpads under the slides were heated to the target temperature for $8 \mathrm{~min}$, then followed with replenishment of CC2 solution every $4 \mathrm{~min}$ if the HD required is $12 \mathrm{~min}$ or longer. The heatpad was disabled and the slide was allowed to cool down in CC2 solution before rinsing with reaction buffer solution and then proceed to the next round of antigen detection.

\section{Staining Reproducibility Study Design and Analysis}

5-Plex Fluorescence IHC (CD3, CD8, CD20, CD68 and FoxP3) staining reproducibility was assessed by three reproducibility studies: (1) intra-day precision study (IDP); (2) inter-day reproducibility intermediate precision study (IDIP) and (3) intra-platform precision study (IPP). In the IDP study, five tonsil cases with replicate slides were stained with one lot of antibody and detection reagents in a single instrument run of 30 slides. Assay staining performance was compared across successive slides from the same case (five study slides and one negative control omitting the $1^{\circ} \mathrm{Ab}$ per case). In the IDIP study, 10 tonsil cases with replicate slides were stained with one lot of antibody and detection reagents and staining performance of the assay was assessed across successive slides from the same cases (two study slides and one negative control without $1^{\circ} \mathrm{Ab}$ per case) over 3 nonconsecutive days. In the IPP study, staining performance of the assay across replicate slides (two study slides and one negative control without $1^{\circ} \mathrm{Ab}$ per case) from 10 tonsil cases using a single lot of antibody and detection reagents was compared among three ULTRA instruments.

5-Plex assay precision was evaluated by intraclass correlation (ICC) or Kendall coefficient of concordance. Mixed effect model based ICC were computed to assess the reproducibility for percentage of positive stained cells. ICC evaluates the reproducibility of quantitative measures between different readings by comparing the variability within group to the variability across group. ICC is between 0 (non-reproducible) and 1 (perfectly reproducible). Kendall coefficient of concordances $\left(K_{\mathrm{w}}\right)$, which is used in a non-parametric method for ordinal data, were computed to evaluate the reproducibility for staining intensity. $K_{\mathrm{w}}$ is between 0 (non-reproducible) and 1 (perfect reproducible).

\section{RESULTS}

\section{The Fluorescent Multiplex Detection Scheme}

As shown in Figure 1, the first on-tissue target is detected by applying target-specific $1^{\circ} \mathrm{Ab}$, then anti-species $2^{\circ} \mathrm{Ab}$ conjugated to HRP, which in turn drives in-situ deposition of Tyfluor. The bound $1^{\circ} \mathrm{Ab}$ and $2^{\circ} \mathrm{Ab}-\mathrm{HRP}$ complex is then deactivated. The deposited Ty-fluor is left covalently bound to tissue near the first target. The same procedure is repeated to detect the next target. This technology allows the use of $1^{\circ} \mathrm{Abs}$ from the same species. To minimize the cross-reactivity, deactivation of bound $1^{\circ} \mathrm{Ab}$ and $2^{\circ} \mathrm{Ab}-\mathrm{HRP}$ complex must result in staining below the detection level in the subsequent round of detection.

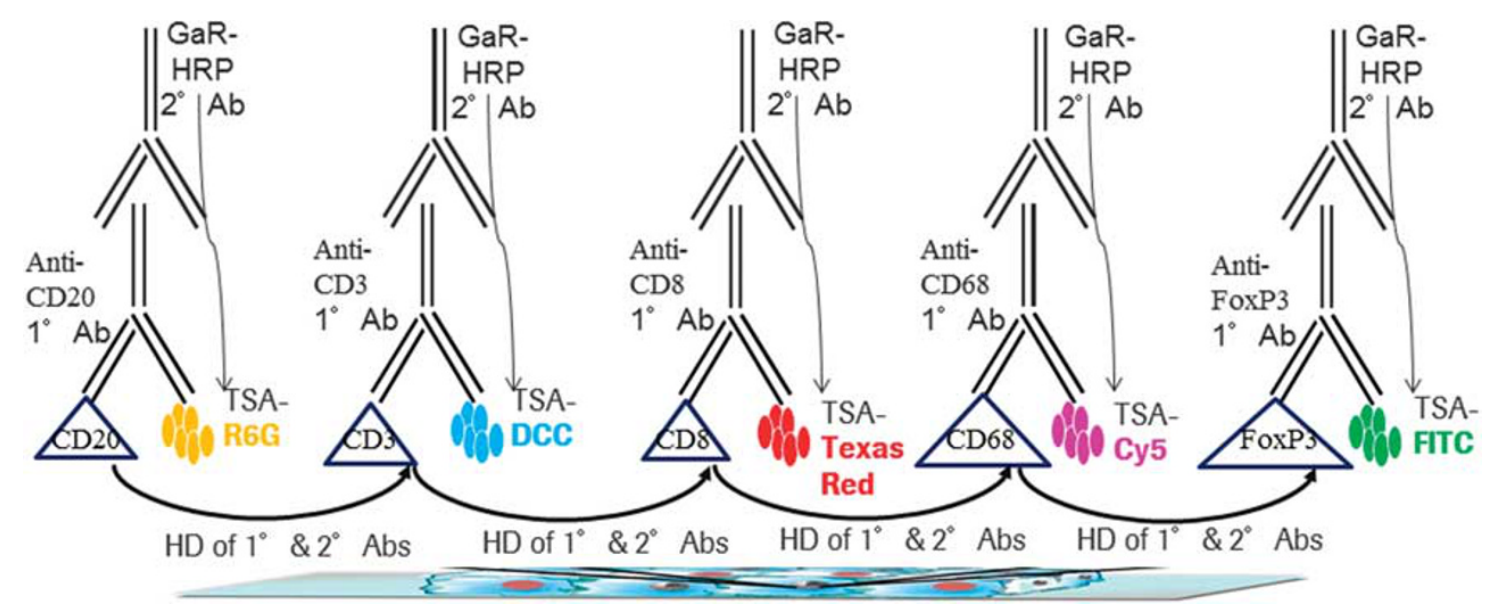

Figure 1 5-Plex IHC detection scheme. 

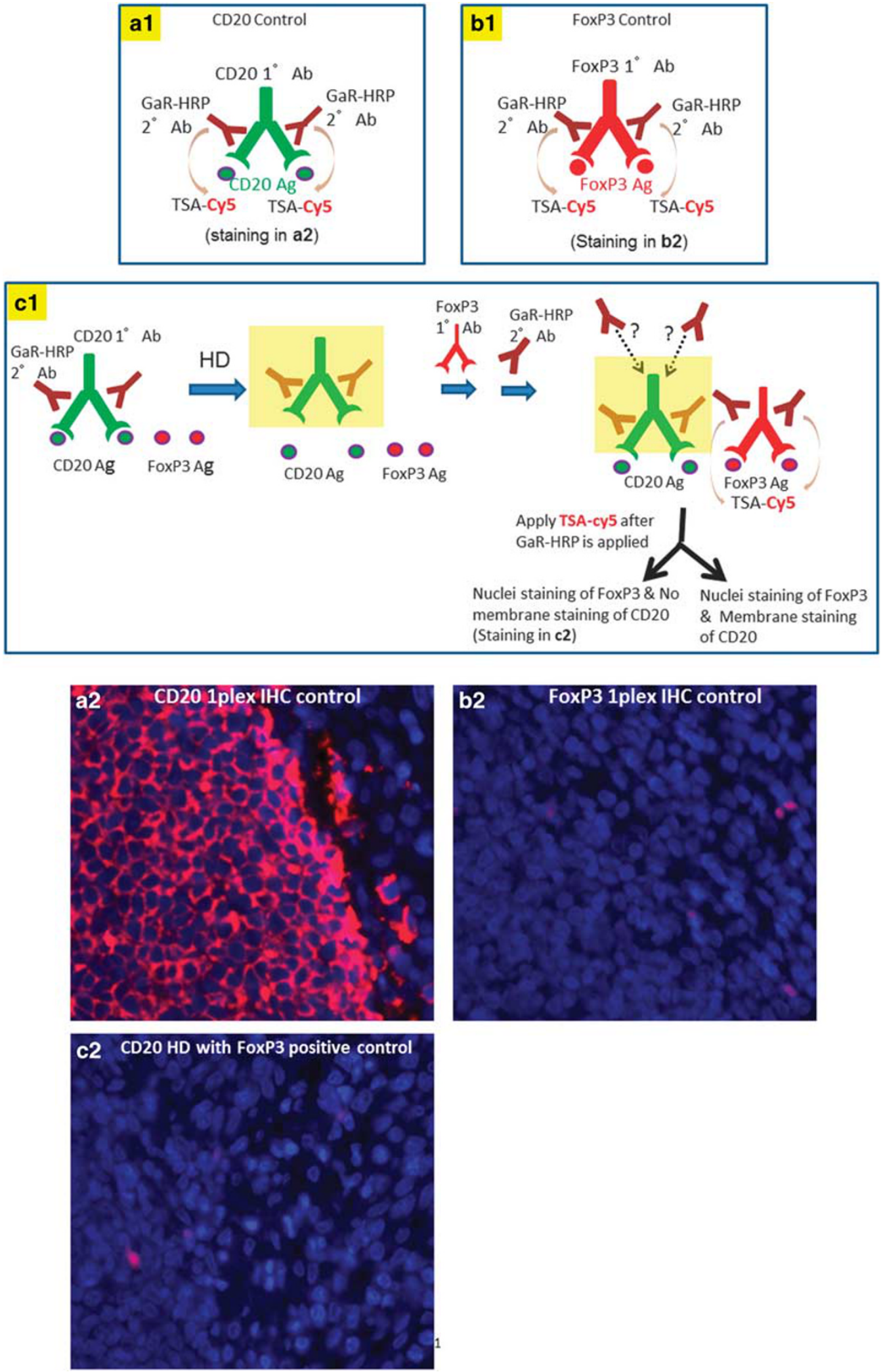

Figure 2 (a1, b1, c1) Experimental design for evaluation of HD effectiveness. (a1) CD20 1-plex IHC control; (b1) FoxP3 1-plex IHC control; (c1) CD20 HD with FoxP3positive control. (a2, b2, c2) Results of CD20 1-plex IHC control staining (a2), FoxP3 1-plex IHC control staining (b2) and CD20 HD with FoxP3-positive control staining. (d-l) Results of CD3 1-plex IHC control staining (d), CD3 HD with FoxP3-positive control staining (e), CD8 1-plex IHC control staining (f), and CD8 HD with FoxP3positive control staining (g), FoxP3 1-plex IHC control staining in the lymphoid tissue area (h), FoxP3 HD with pan-CK-positive control staining in the same area as in $\mathbf{h}$ (i), FoxP3 HD with pan-CK-positive control staining in the epithelium area (j), CD68 1-plex IHC control staining (k), and CD68 HD with FoxP3 positive control staining (I). 

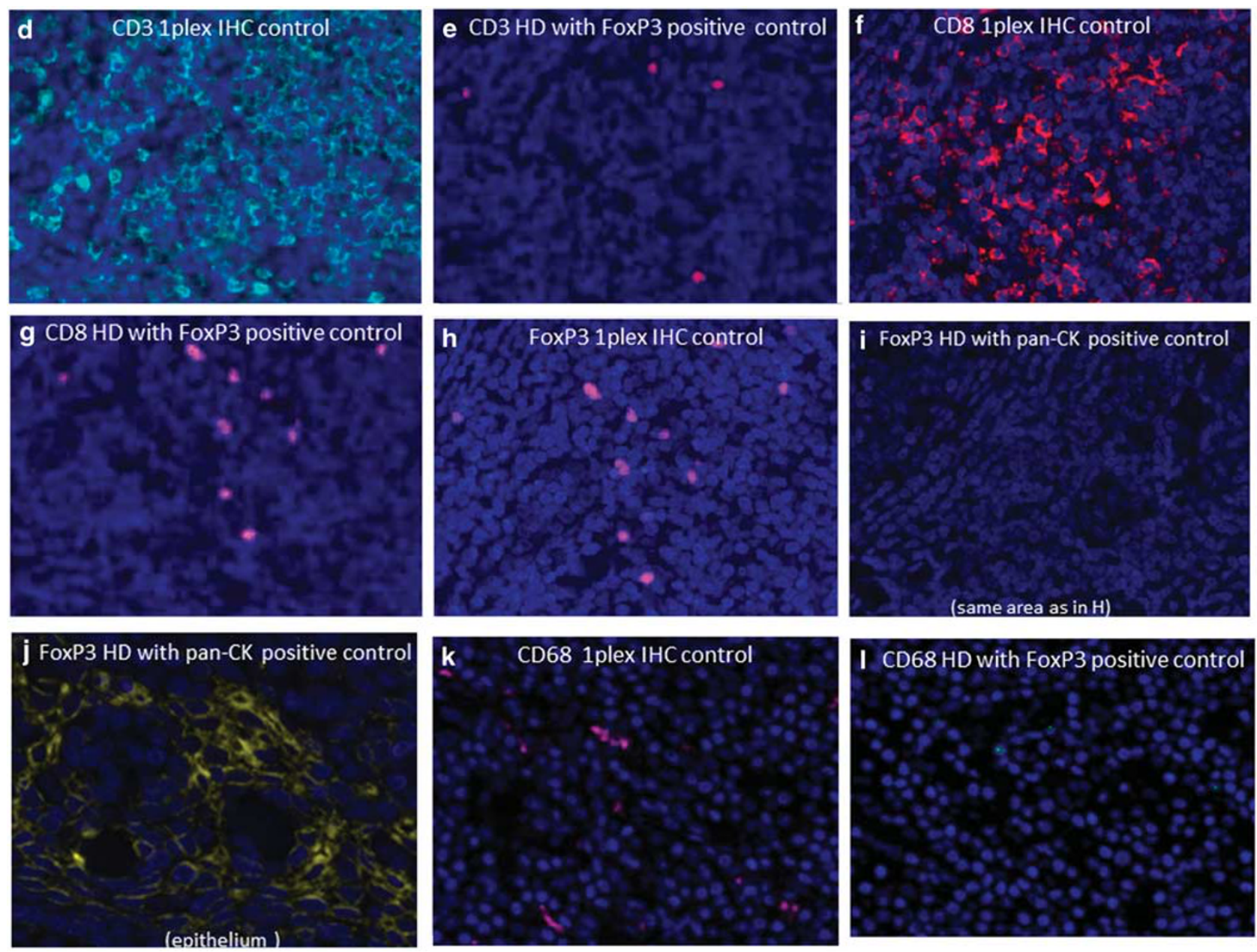

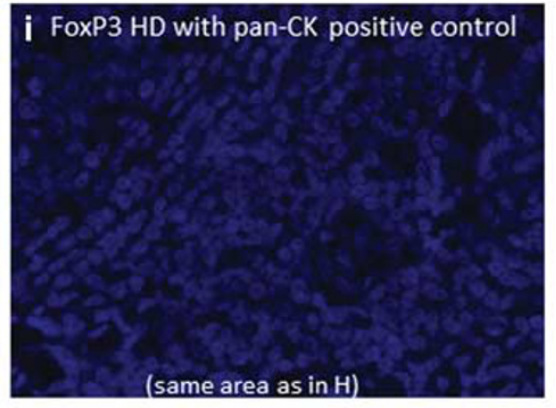

I CD68 HD with FoxP3 positive control

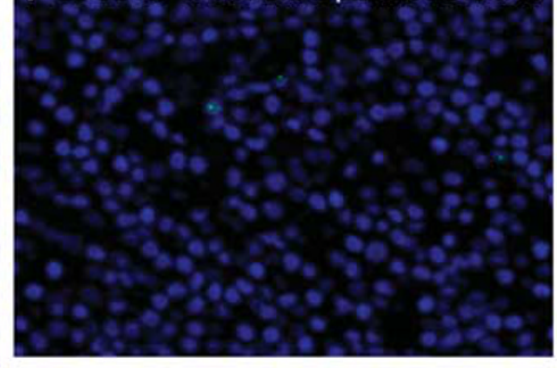

Figure 2 Continued.

\section{Evaluate the Automated HD Using CC2 to Eliminate Cross-Reactivity Between Two Rabbit $1^{\circ} \mathrm{Abs}$ by the Same GaR-HRP $2^{\circ} \mathbf{A b}$ with Tyramide Signal Amplification}

We identified CC2 solution (Ventana cat \# 950-223) at $100{ }^{\circ} \mathrm{C}$ as the most effective deactivation condition on the VENTANA BenchMark ULTRA slide stainer. Figure 2c1 illustrates the experimental design for evaluation of HD effectiveness on $\mathrm{CD} 20 \mathrm{Ab}$ complex: First anti-CD20 $1^{\circ} \mathrm{Ab}$ was applied to the tonsil tissue section on the slide after pretreatment, followed by the application of GaR-HRP $2^{\circ} \mathrm{Ab}$. The tissue section bound with anti-CD20 $1^{\circ} \mathrm{Ab} / \mathrm{GaR}-\mathrm{HRP} 2^{\circ} \mathrm{Ab}$ complex was then treated with $\mathrm{CC} 2$ at $100^{\circ} \mathrm{C}$ for $12 \mathrm{~min}$. Next, the antiFoxP3 $1^{\circ} \mathrm{Ab}$ was applied, followed by the application of the same GaR-HRP $2^{\circ} \mathrm{Ab}$. Last Ty-Cy5 was applied. The GaRHRP bound to anti-FoxP3 $1^{\circ} \mathrm{Ab}$ drives the deposition of Ty$\mathrm{Cy} 5$, which resulted in FoxP3 nuclear staining. If anti-CD20 $1^{\circ} \mathrm{Ab} / \mathrm{GaR}-\mathrm{HRP} 2^{\circ} \mathrm{Ab}$ complex was not deactivated, CD20 membrane staining would also be observed. As shown in Figure 2c2, only FoxP3 nuclear signal but not $\mathrm{CD} 20$ membrane signal was observed, which indicated that the anti-CD20 $1^{\circ} \mathrm{Ab} / \mathrm{GaR}-\mathrm{HRP} \quad 2^{\circ} \mathrm{Ab}$ complex was deactivated below the detection level. As the controls, 1-Plex fluorescence
IHC was conducted for CD20 (Figure 2a1) and FoxP3 (Figure 2b1) in the same instrument run and resulted in the CD20 membrane (Figure 2a2) or FoxP3 nuclear (Figure 2b2) signal respectively. Immunoglobulin (Ig) isotope negative control (negative control rabbit Ig, CONFIRM, cat \# 7601029) was included to confirm specific staining for CD20 1plex IHC control and the CD20 HD study (data not shown). Using similar experiment design, we confirmed the effective $\mathrm{HD}$ below the detection level of CD3 (Figure 2e), CD8 (Figure 2g), FoxP3 (Figure 2i) and CD68 (Figure 2l): FoxP3 was used as the positive staining control in the CD3, CD8 and CD68 HD experiments; keratin detected by anti-Pan keratin (AE1/AE3/PCK26) (cat \# 760-2135) was used as the positive staining control in the FoxP3 HD experiment (Figure 2j). As controls, the 1-plex IHC of CD3 (Figure 2d), CD8 (Figure 2f), CD68 (Figure 2k) and FoxP3 (Figure 2h) showed the expected staining patterns.

\section{Evaluate the Impact of HD on the Fluorophores}

During multiple rounds of IHC in detecting the five markers, the fluorophores covalently bound on the target site at earlier rounds are subjected to subsequent HD steps. To find out the 
a

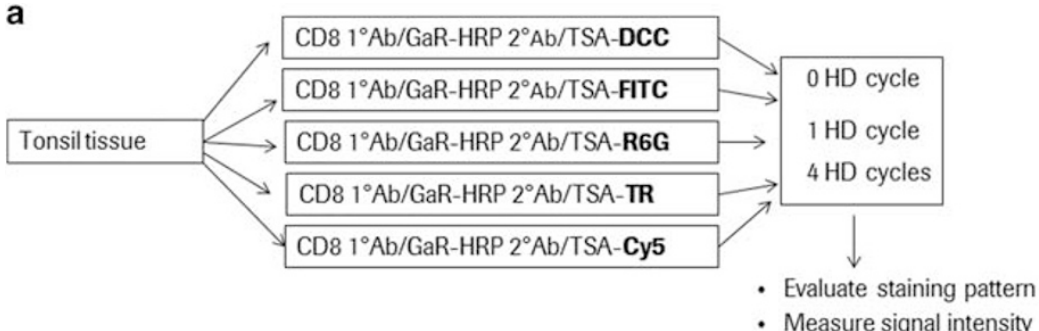

b

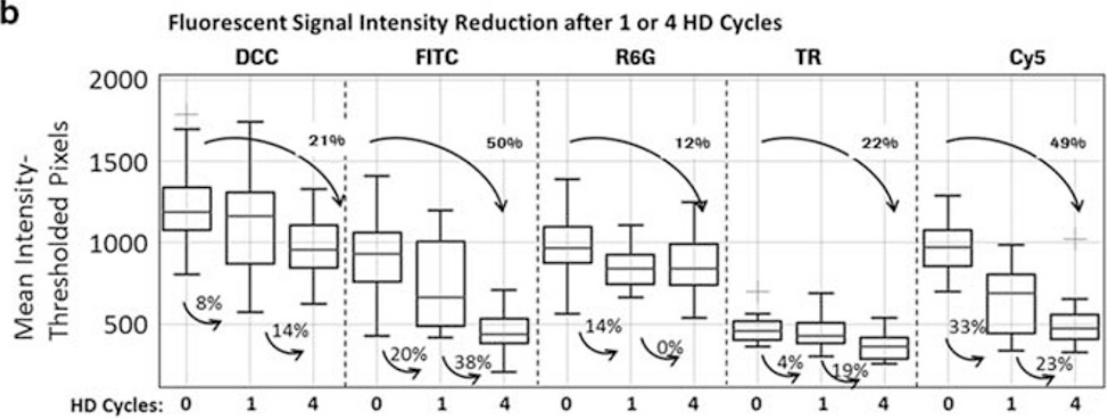

Figure 3 Evaluate the impact of HD on the fluorophores. (a) Study design. (b) Study results.

Tonsil slides

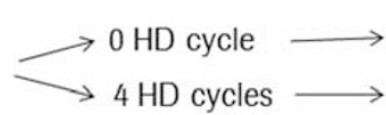

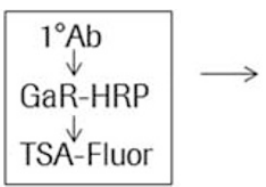

Evaluate staining pattern

and signal intensity

Figure 4 Study design of evaluating the impact of HD on epitope staining.

impact of HD on the deposited fluorophores, we designed the following study on tonsil tissue (Figure 3a). CD8 target antigens were detected with CD8 $1^{\circ} \mathrm{Ab}, \mathrm{GaR}-\mathrm{HRP} 2^{\circ} \mathrm{Ab}$ and each of the five Ty-fluors, followed with one or four cycles of HD. CD8 staining (reported as mean intensity thresholded pixels) was compared with the staining with no HD. Figure $3 \mathrm{~b}$ shows the DCC intensity was reduced $8 \%$ after one cycle of $\mathrm{HD}$, and $21 \%$ after four cycles of HD, FITC intensity was reduced $20 \%$ after one cycle of $\mathrm{HD}$, and $50 \%$ after four cycles of $\mathrm{HD}, \mathrm{R} 6 \mathrm{G}$ intensity was reduced $14 \%$ after one cycle of $\mathrm{HD}$, and $12 \%$ after four cycles of HD, TR intensity was reduced $4 \%$ after one cycle of $\mathrm{HD}$, and $22 \%$ after four cycles of $\mathrm{HD}$, and $\mathrm{Cy} 5$ intensity was reduced $33 \%$ after one cycle of $\mathrm{HD}$, and $49 \%$ after four cycles of HD. Although signal intensity of each Ty-fluor was reduced after one or four cycles of HD, the staining pattern of CD8-positive staining remained unchanged.

\section{Evaluate the Impact of HD on Epitope Staining}

A single antigen retrieval condition and a different number of HD cycles were applied to each target depending on its order in a 5-plex procedure (Figure 4). A study was designed to evaluate the impact of HD cycles on each epitope in 1-Plex IHC by comparing with standard IHC with no HD (Figure 4). Tonsil tissues were stained with the standard IHC

\begin{tabular}{|c|c|c|c|c|}
\hline $\begin{array}{c}\text { Detection } \\
\text { order }\end{array}$ & TSA Fluor & $\begin{array}{c}\text { Effect of HD } \\
\text { on Fluor }\end{array}$ & $1^{\circ} \mathrm{Ab}$ & $\begin{array}{c}\text { Effect of HD } \\
\text { on Epitope }\end{array}$ \\
\hline 1 & R6G TSA & Les Affected & CD20 & \begin{tabular}{c} 
Most Affected \\
\hline 2
\end{tabular} \\
\hline DCC TSA & & & CD3 & \\
\hline 3 & Texas Red TSA & & CD8 & \\
\hline 4 & Cy5 TSA & & CD68 & \\
\hline 5 & FITC TSA & MostAeffected & FoxP3 & \\
\hline
\end{tabular}

Figure 5 5-Plex IHC assay configuration.

processes including $100{ }^{\circ} \mathrm{C}$ for $64 \mathrm{~min}$ antigen retrieval, followed with either no HD or four cycles of $\mathrm{HD}$ $\left(10{ }^{\circ} \mathrm{C}\right.$ for $12 \mathrm{~min}$ each). Each epitope (CD3, CD8, CD20, CD68 or FoxP3) was detected with their respective $1^{\circ} \mathrm{Ab}$, GaR-HRP $2^{\circ} \mathrm{Ab}$ and Ty-R6G. Slides stained with no HD or four cycles of HD were evaluated under the fluorescence microscopy for signal intensities $(0,1,2$ and 3$)$. There was a slight reduction of signal intensity observed for CD3 (from 3 to 2.5) and CD20 (from 2.5 to 2) after four HD cycles, comparing with no HD; no signal intensity reduction was 
a

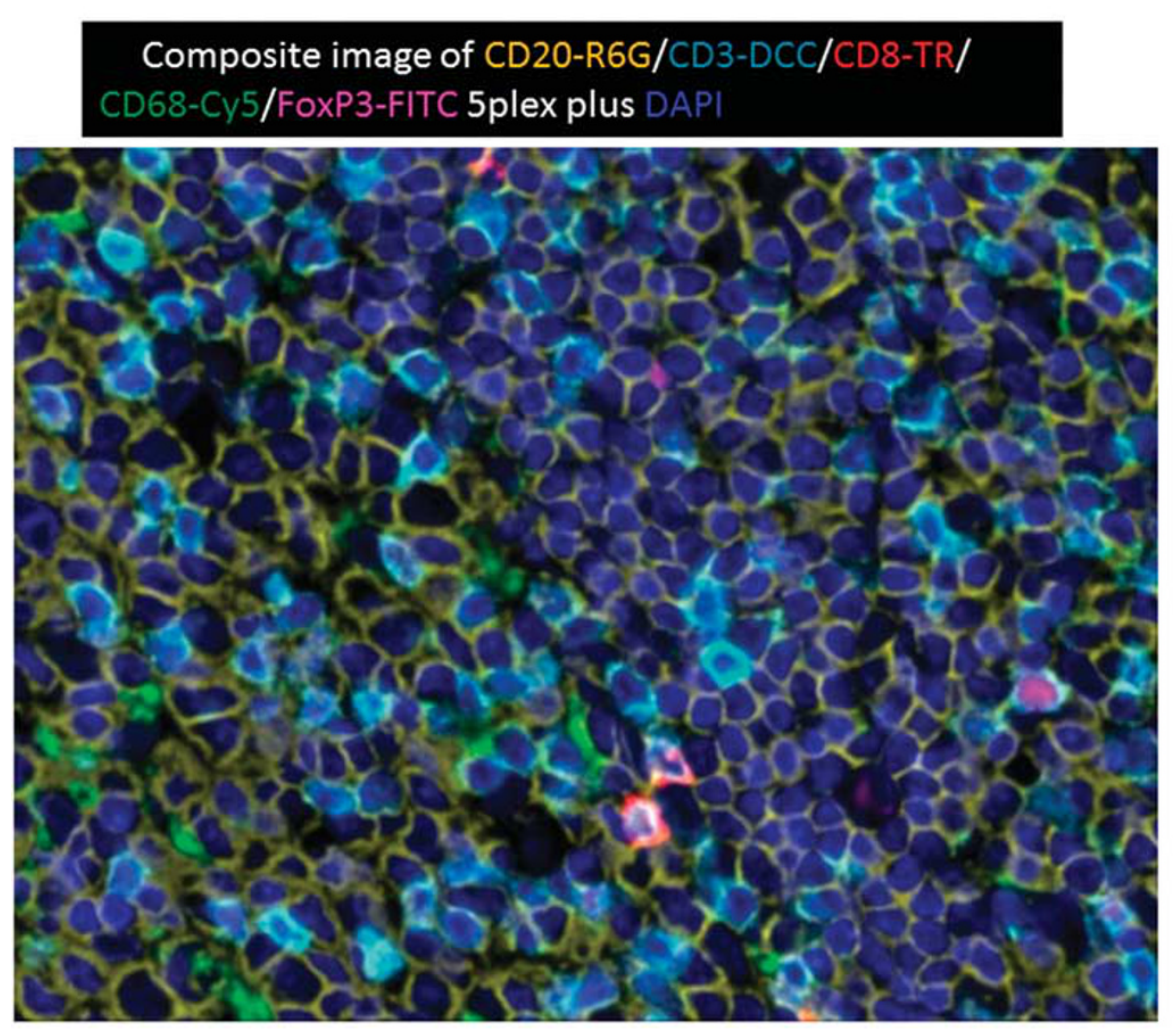

b
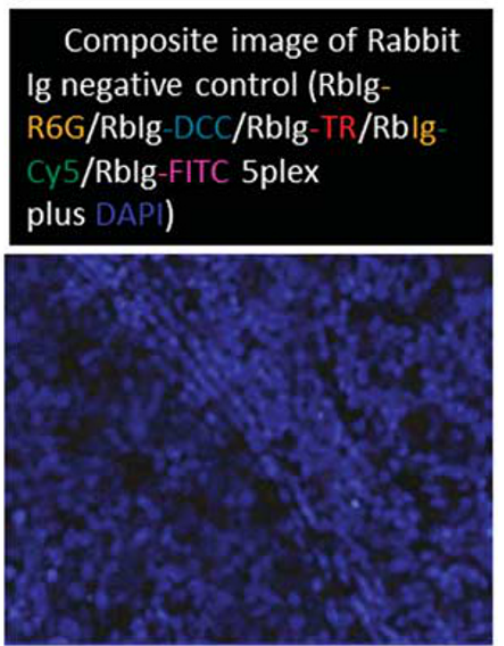

\begin{tabular}{|c|c|}
\hline Channel & Pseudo Color \\
\hline CD20 (or Rblg)-R6G & Yellow \\
\hline CD3 (or Rblg)-DCC & Cyan \\
\hline CD8 (or Rblg)-TR & Red \\
\hline CD68 (or Rblg)-Cy5 & Green \\
\hline FoxP3 (or Rblg)-FITC & Magenta \\
\hline DAPI & Blue \\
\hline
\end{tabular}

C

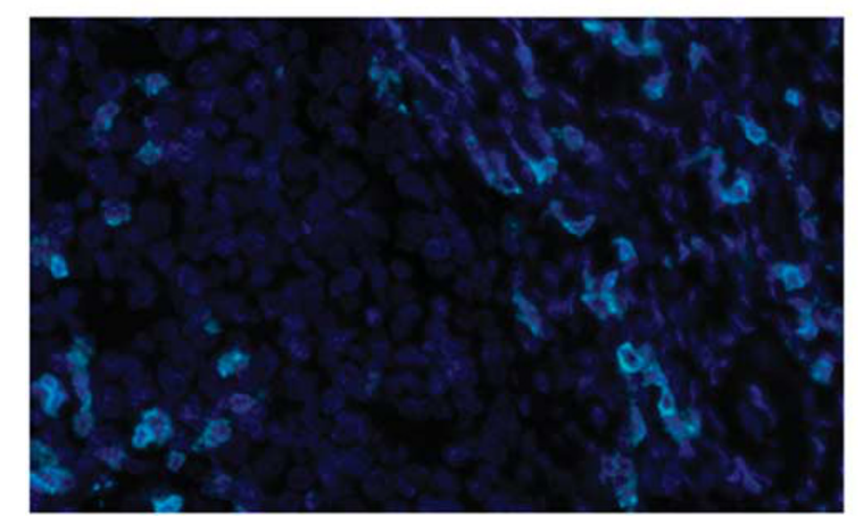

d Rblg-TR in 5plex (TR + DAPI channels)

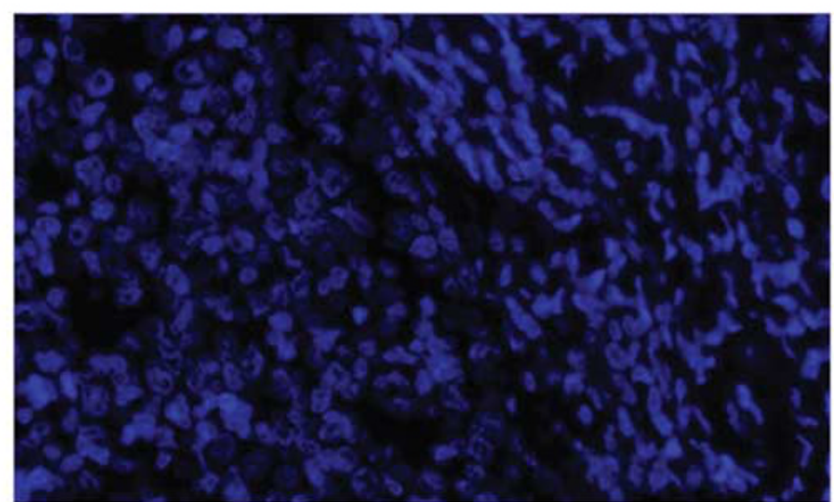

Figure 6 Representative CD20/CD3/CD8/CD68/PoxP3 5-plex plus DAPI staining images on tonsil tissues. (a) Composite image of CD20-R6G with yellow pseudocolor, CD3-DCC with cyan pseudocolor, CD8-TR with red pseudocolor, CD68-Cy5 with green pseudocolor, FoxP3-FITC with magenta pseudocolor, DAPI with blue pseudocolor, $40 \times$. (b) Composite image of negative control rabbit lg (ie, Rblg) (Rblg-R6G/Rblg-DCC/Rblg-TR/Rblg-Cy5/Rblg-FITC plus DAPI, $40 \times$. (c) Composite image of CD3/DCC and DAPI of the CD20/CD3/Rabbit IgG/CD68/PoxP3 5-plex IHC. (d) Composite image of Rb IgG/TR and DAPI of the CD20/CD3/rabbit lgG/CD68/PoxP3 5-plex IHC.

observed for CD8 (intensity 2), CD68 (intensity 3) and FoxP3 (intensity 3).

\section{5-Plex IHC Assay Configuration}

Based on the above evaluations, we decided to place the Ty-fluors that are impacted the least by HD in the earlier IHC rounds, and use those Ty-fluors that are impacted the most in the later IHC rounds (Figure 5). Similarly, we detected the epitopes more sensitive to HD in an early IHC round, and the epitopes less sensitive to HD in the latter IHC rounds (Figure 5). The 5-plex IHC configuration is shown in Figure 1: CD20 and Ty-R6G in the first IHC round, CD3 and Ty-DCC in the second IHC round, CD8 and Ty-TR in the third IHC round, CD68 and Ty-Cy5 in the fourth IHC 
Table 2 ICC for percentage of positive cells and $K_{\mathrm{w}}$ for staining intensity

\begin{tabular}{lllllll}
\hline & Precision & CD20 & CD3 & CD8 & CD68 & FoxP3 \\
\hline ICC for percentage of & IDIP & 0.95 & 0.90 & 0.89 & 0.74 & 0.88 \\
positive stained cells & IDP & 0.95 & 0.93 & 0.46 & 0.89 & 0.85 \\
& IPP & 0.95 & 0.85 & 0.74 & 0.87 & 0.87 \\
$K_{\mathrm{w}}$ for staining intensity & IDIP & 1.00 & 0.78 & 0.87 & 1.00 & 1.00 \\
& IDP & 1.00 & 0.96 & 0.45 & 1.00 & 0.90 \\
& IPP & 1.00 & 0.40 & 0.41 & 1.00 & 1.00 \\
\hline
\end{tabular}

round and FoxP3/FITC in the fifth IHC round. The DAPI counterstain is then performed. Figure $6 \mathrm{a}$ shows a representative composite image of CD20/CD3/CD8/CD68/FoxP3 5 -plex IHC plus DAPI on a tonsil tissue (pseudocolors were used for image viewing purpose, not reflecting the fluorescent colors viewed under a microscope). Figure $6 \mathrm{~b}$ shows a representative composite image of 5-plex IHC staining with negative control rabbit Ig plus DAPI counterstain. CD8+ cells are a sub-population of CD3+ cells. To demonstrate that the CD8+ staining was specific signal and not resulted from incomplete $\mathrm{HD}$ of $\mathrm{CD} 3$ antibody complex, we conducted CD20/CD3/rabbit IgG/CD68/FoxP3 5-plex IHC in which the anti-CD8 antibody was replaced with rabbit IgG. Only CD3+ cells but no CD8+ staining was observed. Figure $6 \mathrm{c}$ shows a representative image of CD3-DCC plus DAPI; Figure 6d shows a representative image of rabbit IgG-TR plus DAPI.

\section{5-Plex Assay Staining Reproducibility Evaluated on Tonsil}

Both percentages of positive stained cells and staining intensities were evaluated by the pathologist (L Zhang), and demonstrated consistency and reproducability (Table 2). For readout of percentage of positive stained cells, markers CD20, CD3 and FoxP3 showed excellent reproducibility in both IDIP and IPP with ICC values $>0.85$. Marker CD68 showed excellent reproducibility in both IDP and IPP (ICC >0.85), and good reproducibility in IDIP (ICC $=0.74)$. Marker CD8 showed excellent reproducibility in IDIP (ICC $=0.89$ ), good reproducibility in IPP $(\mathrm{ICC}=0.74)$, and fair reproducibility in IDP $($ ICC $=0.46)$. For staining intensity, marker CD20, CD68 and FoxP3 showed excellent reproducibility in all three studies $\left(K_{\mathrm{W}}>0.90\right)$. Marker CD8 showed excellent reproducibility in IDIP $\left(K_{\mathrm{W}}=0.87\right)$ and fair reproducibility in both IDP and IPP. Marker CD3 showed excellent reproducibility in IDP $\left(K_{\mathrm{w}}=0.96\right)$, good reproducibility in IDIP $\left(K_{\mathrm{w}}=0.78\right)$ and fair reproducibility in IPP $\left(K_{\mathrm{w}}=0.40\right)$.

\section{Equivalency of the 5-Plex FL Assay Staining with the Single IHC DAB Staining on Tonsil and Tumor Specimens} Comparison of 5-plex fluorescent staining with single IHC $\mathrm{DAB}$ staining was conducted on six successive tonsil tissue slides, in which CD20 DAB was stained on the first slide, CD3 $\mathrm{DAB}$ on the second slide, CD8 DAB on the third slide, 5-plex on the fourth slide, CD68 DAB on the fifth slide and FoxP3 $\mathrm{DAB}$ on the sixth slide. The whole-slide images were obtained from the stained slides scanned on a Zeiss Axioscan.Z1 slide scanner. The staining pattern of individual DAB-stained slides were compared with each channel of the 5-plex fluorescent slide by the pathologist (L Zhang) using split-view images in Zen Blue software (Figure 7a). The split image viewing function allowed the viewing of the corresponding tissue area in both fluorescent and DAB images side by side. Both images were zoomed in and out and moved around simultaneously to help to compare staining pattern and percentage of positive stained cells. All the five markers showed comparable staining patterns (subcellar locations and percentage positive cells) between fluorescent 5-plex and single DAB (see the example images in Figure 7a). For each of the five markers, the percentage of cells with positive staining from the same area on the fluorescent slide and the corresponding single DAB slide was estimated by the pathologist (L Zhang): CD3: 35\% (fluorescent) vs 30\% (DAB); CD8: 15\% for both; CD20: 95\% for both; CD68: 95\% for both; FoxP3: 4\% (fluorescent) vs 3\% (DAB).

Similar evaluations were performed on a multitumor tissue array slide containing 48 cases of 15 cancer types. Comparable 5-plex fluourescent to single DAB staining was observed in all the tissue cores. The representative images are shown in Figure $7 \mathrm{~b}$ (ovarian carcinoma), Figure 7c (breast tumor) and Figure $7 \mathrm{~d}$ (hepatocellular carcinoma).

\section{DISCUSSION}

Our study results show for the first time the feasibility of an automated multiplex fluorescent IHC with tyramide signal amplification using $1^{\circ} \mathrm{Abs}$ from the same species. A major advantage of the present work over previously described manual or semi-manual $\mathrm{mIHC}$ is the deactivation process of $1^{\circ} \mathrm{Ab} / 2^{\circ} \mathrm{Ab}$ immunocomplex achieved on an automated slide staining platform. We found this method was effective and reproducible on a variety of protein markers, the respective $1^{\circ}$ Abs, and tissue types. We successfully applied this method to a mIHC assay for a panel of immunomarkers consisting CD3, CD8, CD20, CD68 and FoxP3 on tonsil tissues.

The unwanted cross-reactivity between $1^{\circ} \mathrm{Ab}$ and $2^{\circ} \mathrm{Ab}$ from different staining rounds is regarded as the main technical risk in mIHC. ${ }^{24,25}$ Specificity of $2^{\circ} \mathrm{Abs}$ is generally based on the species used to raise $1^{\circ} \mathrm{Ab}$ and $\mathrm{Ig}$ class or subclass. As most of the commercially available Abs are raised either in mouse or rabbit as IgG1, it is a common problem that Abs of interest are from the same species. The most reported solution is a manual processes that targets $1^{\circ} \mathrm{Ab} / 2^{\circ}$ $\mathrm{Ab}$ immunocomplexes in the preceding round by microwaving the tissue in $10 \mathrm{mM}$ citrate buffer, $\mathrm{pH}$ 6.0, before incubation with another $1^{\circ} \mathrm{Ab}$ from the same species and the same anti-species $2^{\circ} \mathrm{Ab}$ in the subsequent round. Lan et $a l^{4}$ reported microwaving slides in $10 \mathrm{mM}$ citrate buffer, $\mathrm{pH} 6.0$ to the boiling point twice for $5 \mathrm{~min}$ to deactivate a mouse 
a
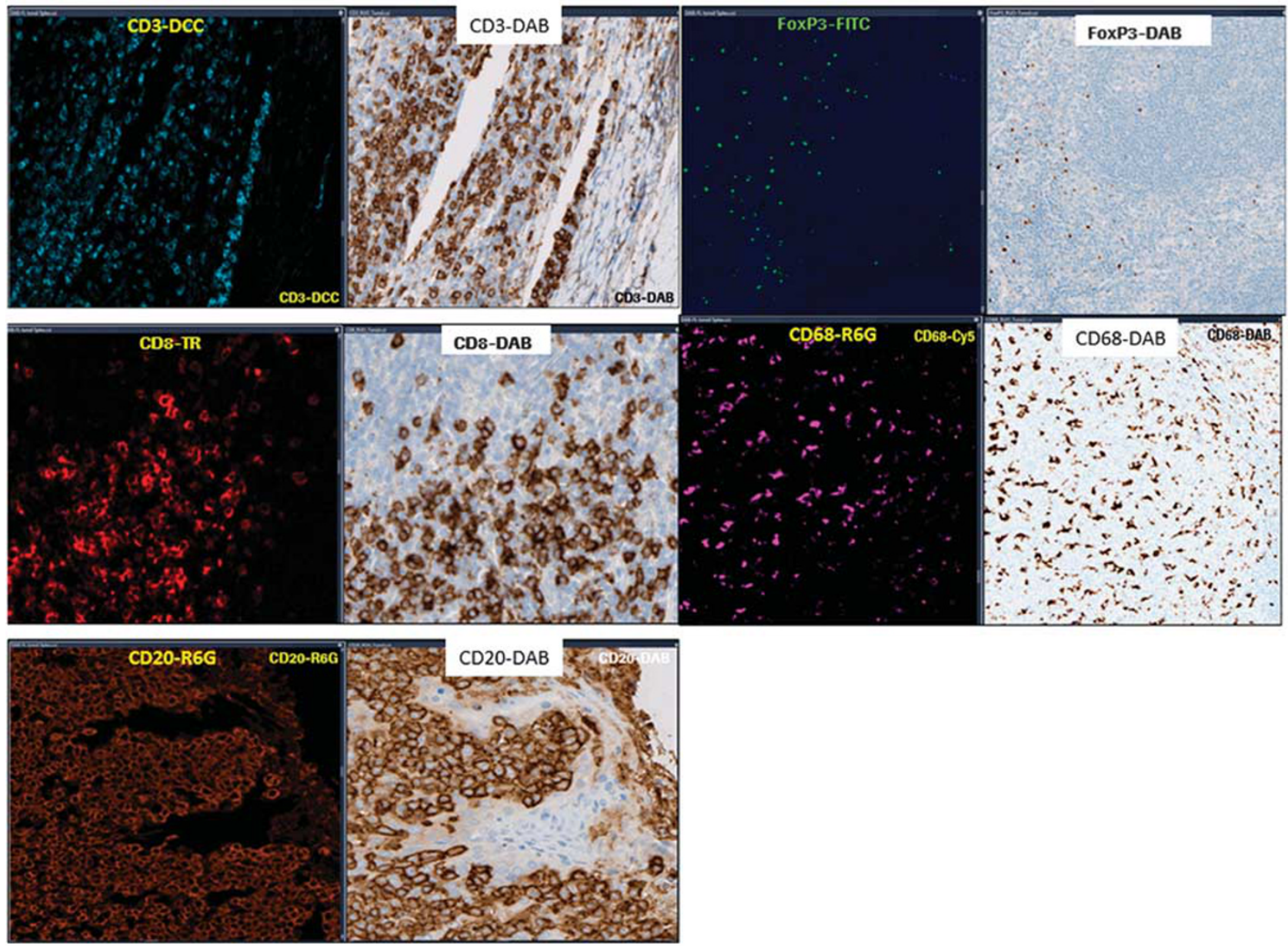

b
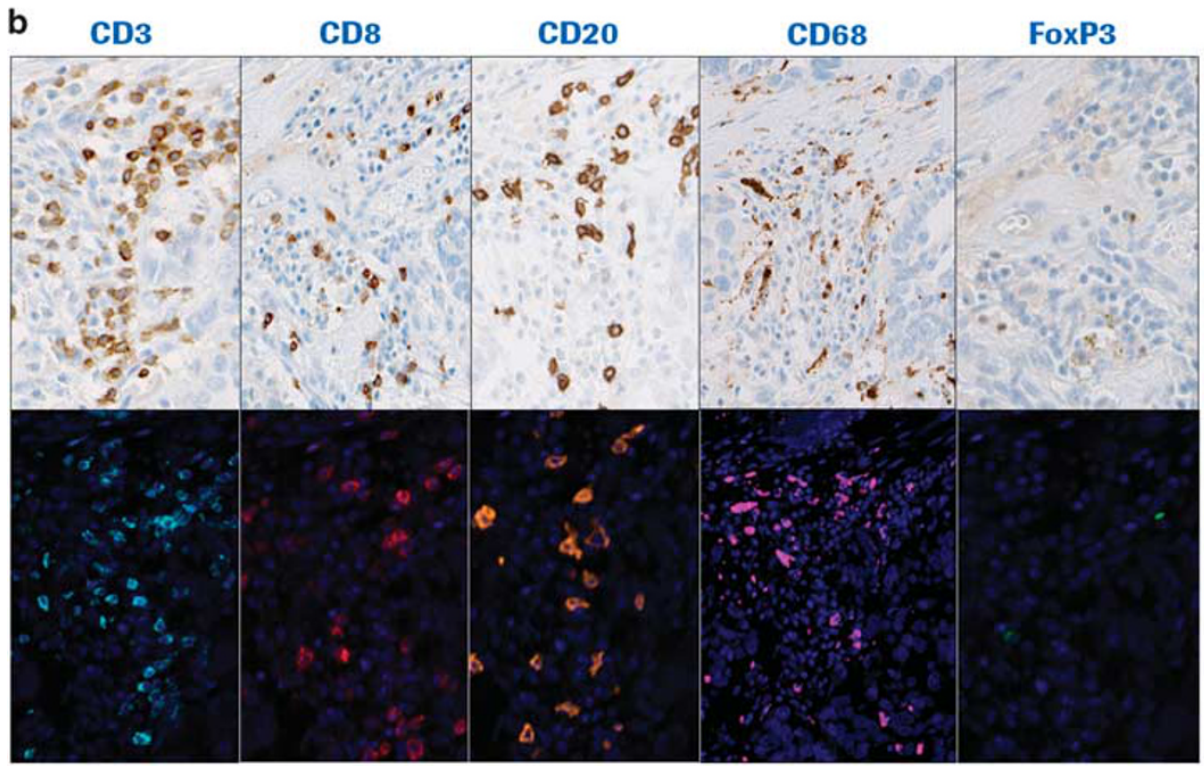

DCC

Texas Red

R6G

Cy5

FITC

Figure 7 (a) Comparable staining between 1-plex DAB and 5-plex FL on tonsil. (b) Comparable staining between 1-plex DAB and 5-plex FL on ovarian tumor. (c) Comparable staining between 1-plex DAB and 5-plex FL on breast tumor. (d) Comparable staining between 1-plex DAB and 5-plex FL on liver tumor. 

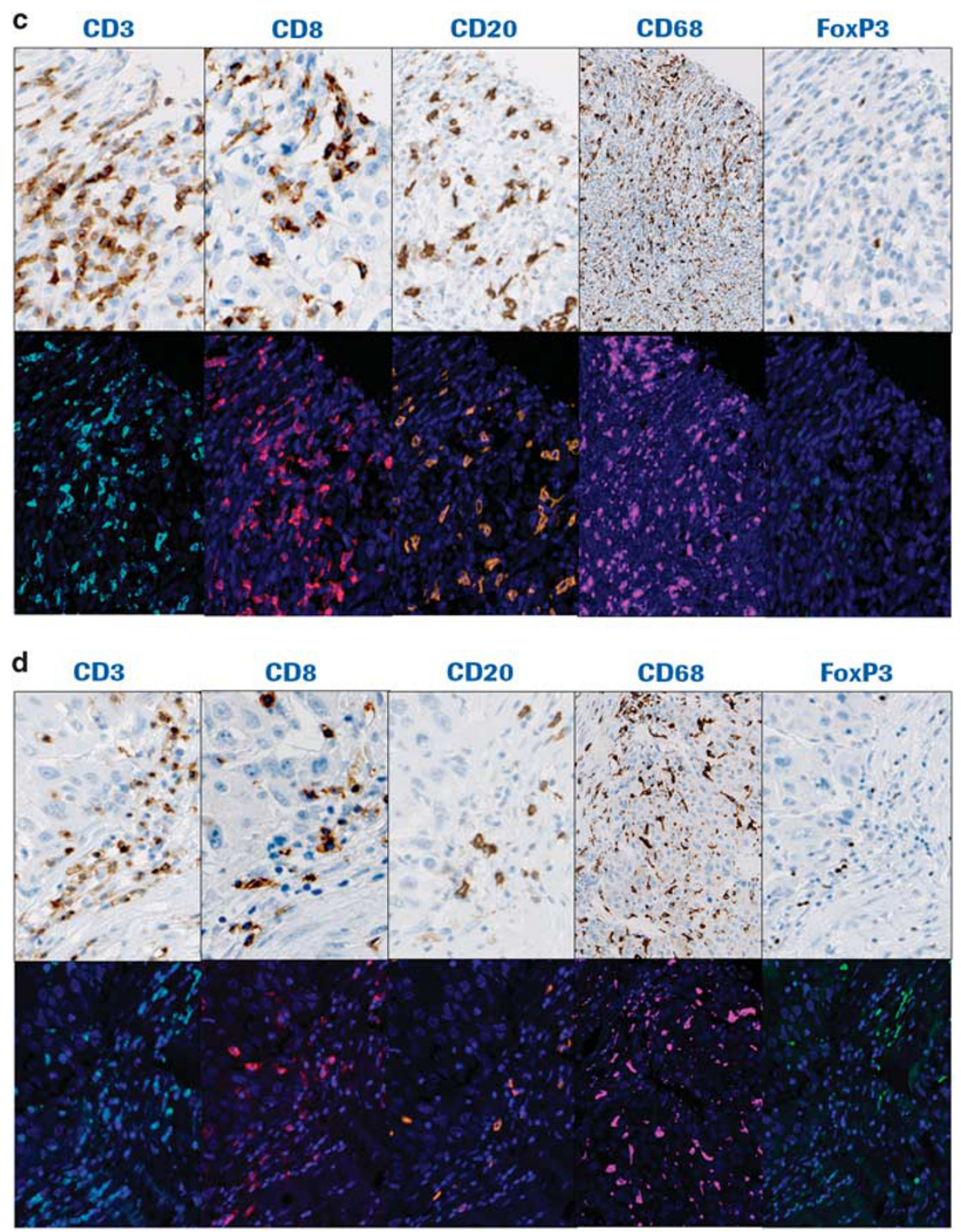

Figure 7 Continued.

anti-rat PC-10 $1^{\circ} \mathrm{Ab}$ and mouse anti-human CD3 and CD68 $1^{\circ} \mathrm{Abs}$ on rat and human tissues with chromogenic detection. Bauer et al ${ }^{6}$ tested microwaving in $10 \mathrm{mM}$ citrate buffer, $\mathrm{pH}$ 6.0 for $5 \mathrm{~min}$ three times, but failed to deactivate mouse antiASM-1 $1^{\circ} \mathrm{Ab}$ on bovine ovaries with chromogenic detection. Tornehave et al ${ }^{5}$ reported microwaving in $10 \mathrm{mM}$ citrate buffer, $\mathrm{pH} 6.0$ for $5 \mathrm{~min}$ three times to deactivate a rabbit anti-somatostatin $1^{\circ} \mathrm{Ab} / 2^{\circ} \mathrm{Ab}$ complex, mouse anti-glucagon $1^{\circ} \mathrm{Ab} / 2^{\circ} \mathrm{Ab}$ complex and mouse anti-FSH $1^{\circ} \mathrm{Ab} / 2^{\circ} \mathrm{Ab}$ complex in a 2-plex fluorescence IHC with anti-species $2^{\circ} \mathrm{Ab}$ fluorphore conjugates on rat and mice tissues. Tóth and Mezey $^{7}$ microwaved in $10 \mathrm{mM}$ citrate buffer, $\mathrm{pH} 6.0$ to the boiling point for 2.5-min and 5-min periods to deactivate (1) mouse anti-NeuN $1^{\circ} \mathrm{Ab} / 2^{\circ} \mathrm{Ab}$ complex in a 2-plex fluorescence IHC with Ty-fluor conjugates, and (2) rabbit anti-VP $1^{\circ}$ $\mathrm{Ab} / 2^{\circ} \mathrm{Ab}$ complex and rabbit anti- $\mathrm{TH} 1^{\circ} \mathrm{Ab} / 2^{\circ} \mathrm{Ab}$ complex in a 3-plex fluorescence IHC with Ty-fluor conjugates on mouse and rat tissues. Osman et al ${ }^{8}$ reported microwaving in $\mathrm{pH} 6.0$ Target Retrieval Buffer (Dako) at $74^{\circ} \mathrm{C}$ for $7.5 \mathrm{~min}$ to deactivate (1) mouse anti-P75NGFR $1^{\circ} \mathrm{Ab}$ complex and mouse antiALDH $11^{\circ} \mathrm{Ab}$ complex, and (2) mouse anti-BMI1 $1^{\circ} \mathrm{Ab}$ complex and mouse anti-P75NGFR $1^{\circ} \mathrm{Ab}$ complex in a 3 -plex IHC assay with chromogenic detection on human tissues. Besides the microwaving methods, Suzuki et a ${ }^{26}$ reported that heating slides in $10 \mathrm{mM}$ citrate buffer, $\mathrm{pH} 6.0$ at $90^{\circ} \mathrm{C}$ for $15 \mathrm{~min}$ deactivated (1) guinea pig anti-insulin $1^{\circ} \mathrm{Ab}$ complex and rabbit antiglucagan $1^{\circ} \mathrm{Ab} / 2^{\circ} \mathrm{Ab}$ complex, (2) mouse anti-CD4 $1^{\circ} \mathrm{Ab}$ complex and mouse anti-CD8 $1^{\circ} \mathrm{Ab}$ complex in a 3 -plex fluorescence IHC with biotin/streptavidin-fluorophore and antispecies $2^{\circ} \mathrm{Ab}$-fluorophore conjugate detection on human tissues.

Although microwaving is a useful research tool in $\mathrm{mIHC}$ using $1^{\circ} \mathrm{Abs}$ from the same species, it may not be an ideal 
method to be adopted in clinical in vitro diagnostic (IVD) assays. The different methods and results described above imply a variable manual process. Moreover, microwaving is known to increase the tendency of tissue damages. Our present work takes advantage of existing antigen retrieval mechanisms (ie, buffers and temperatures) on a clinical automated slide stainer, and therefore, enables a reliable and practical HD solution for $\mathrm{MIHC}$ as a potential IVD assay. Automation standardizes HD and other critical IHC steps, that is, control of reagent application and washing steps, kinetics optimization, control of temperatures, control of evaporation and humidity, etc. ${ }^{27-29}$ Our study results indicate this optimized automated system allows accurate, consistent and reproducible mIHC staining using same species $1^{\circ} \mathrm{Abs}$.

Thermal denaturation of IgG, a complex process involving IgG unfolding and aggregation, mainly depends on $\mathrm{pH}$, temperature and solution conditions. ${ }^{30,31}$ The Fab fragment is most sensitive to heat treatment, whereas the Fc fragment is most sensitive to decreasing $\mathrm{pH}$. Heat-induced studies on whole IgG showed a $50 \%$ denaturation of the Fab and Fc domains at $61^{\circ} \mathrm{C}$ and $71{ }^{\circ} \mathrm{C}$, respectively. ${ }^{30}$ Studies also suggest formation of a $1^{\circ} \mathrm{Ab} / 2^{\circ} \mathrm{Ab}$ complex stabilizes the structure, therefore higher heating condition may be required for deactivation. Studies on $\mathrm{pH}$-dependent peptide bond fragmentation suggest the reaction rate is minimal around neutral $\mathrm{pH}$ and is accelerated under acidic and basic conditions. ${ }^{31}$ Other factors, such as low molecular weight surfactants and the denaturing surfactant SDS lead to a lower thermal stability. ${ }^{32}$ High surfactant concentration may help prevent aggregation of denatured IgG, and thus improve the removal of heatdeactivated proteins. We found many antibodies that were satisfactorily deactivated in HD step with lower temperatures $\left(80-90^{\circ} \mathrm{C}\right)$ (data not shown) but all Abs were successfully HD with $100 \mathrm{C}$ (actual/recorded $97^{\circ} \mathrm{C}$ ) for $12 \mathrm{~min}$ in CC2.

To build a mIHC assay using sequential detection with unmodified $1^{\circ} \mathrm{Ab}$ and TSA-fluor detection, we first optimized assay conditions for appropriate staining. For common reagents, such as Ty-fluors, anti-species-HRP conjugates (ie, GaR-HRP), and $\mathrm{H}_{2} \mathrm{O}_{2}$, we locked down the optimal configurations suitable to detecting different $1^{\circ} \mathrm{Abs}$ for optimal signal/background ratio. The order of Ty-fluor deposition is determined by the effect of $\mathrm{HD}$ on each fluor, that is, the least $\mathrm{HD}$ affected fluor deposited first and the most affected fluor deposited last. For $1^{\circ} \mathrm{Abs}$, we determined the concentrations that provided optimal signal/background ratio for a broad dynamic range of protein expression levels on different tissue types. The order of $1^{\circ} \mathrm{Ab}$ deposition was determined by the effect of HD on each epitope, that is, the most HD affected epitope detected first, the least affected epitope detected last. For example, we determined the order of $1^{\circ} \mathrm{Ab}$ deposition for the CD20/CD3/CD8/CD68/FoxP3 5 -plex by evaluating the effect of $\mathrm{HD}$ on each of the target epitope using human tonsil tissue. The same antibody deposition sequence resulted in appropriate 5-plex staining in multiple tissue types tested. It is important to perform such evaluation on the tissue types of interest when optimizing an assay for testing clinical samples.

We further optimized staining performance by varying other assay parameters, that is, optimizing epitope retrieval time to balance signal, background and tissue morphology, optimizing $1^{\circ} \mathrm{Ab}$ incubation time, $2^{\circ} \mathrm{Ab}$ incubation time and TSA reaction time to offer the best detection sensitivity. With the above efforts, an optimal mIHC assay should achieve the following staining performances: (1) comparable positive staining pattern/percentage to single DAB IHC staining, (2) robustness to stain a dynamic range of low and high target levels, (3) desirable subcellular staining patterns: that is, circumferential membrane staining allowing individual cell boundaries delineation for membrane markers, staining restricted to the intracellular structures for cytoplasmic and nuclear markers, (4) minimal bleed through to adjacent channels. We carefully chose the fluorophores and designed the narrow-band filters to minimize bleed through. Next, we validated an effective HD condition on the intended tissues after having achieved desirable staining performance. In our study, the optimal $1^{\circ} \mathrm{Ab}$ concentrations in Ty-Fluor detection are within a twofold range of that used in $\mathrm{DAB}$ detection. This is distinct from Hunyady B 1997's method, where $1^{\circ} \mathrm{Abs}$ were diluted at a very low (ie, 10-fold) concentration. We do not recommended a HD condition, which is only effective with extremely low $1^{\circ} \mathrm{Abs}$ concentration and/or suboptimal epitope retrieval condition as it compromises assay robustness.

A major technical risk of this approach is that insufficient deactivation of the $1^{\circ} \mathrm{Ab} / 2^{\circ} \mathrm{Ab}$ complexes may cause crossreaction and potentially resulting in false-positive signals. Besides efforts to identify proper HD during assay development, we may also take advantage of imaging tools to resolve potential cross-reaction. For non-colocalized markers, image analysis can detect and remove the false-positive staining. For two markers, where one is the sub-population of the other, we can design the assay to detect the sub-population first so cross-reaction will have no impact on staining interpretation. For two markers that do not always co-express, we can design an assay to place a non-colocalized marker in between. The cross-reactivity will be detected by imaging analysis, and the slide will be disqualified. A new slide should then be run under harsher HD condition.

Higher level (eg, >7) multiplexing solutions have been emerging in recent years. Gerdesa ${ }^{33}$ and Sood $^{34}$ described 'MultiOmyx' multiplexing that offers sequential staining of up to 60 proteins using fluorophore-conjugated $1^{\circ} \mathrm{Abs}$ and a 'stain-erase' procedure that chemically inactivates the fluorophores that allows tissue slides to be reused. This imagingbased technology relies on the assumption that the cellular architecture remains sufficiently intact to allow image coregistration between different staining cycles. The directly labeled $1^{\circ} \mathrm{Abs}$ without signal amplification is also vulnerable for detection robustness to low abundant targets. The limited area of analysis represents a major challenge to translate it into a practical and economical pathology workflow. Angelo ${ }^{35}$ 
described multiplexed ion beam imaging that uses secondary ion mass spectrometry to image $1^{\circ} \mathrm{Abs}$ tagged with isotopically pure elemental metal reporters, capable of analyzing up to 100 targets simultaneously. Imaging instrument throughput is an obstacle to broader application of this method. Using conjugated $1^{\circ} \mathrm{Abs}$ for protein detection, this technology inherits many of the issues that can limit the utility of conventional IHC. Although the methodologies are complex, the potential for mass spectroscoy is developing greater practical demonstration.

In conclusion, this technology enables an automated mIHC assay through the use of commercially available native $1^{\circ} \mathrm{Abs}$ with their respective anti-species $2^{\circ} \mathrm{Ab}$, and a clinically proven staining platform to ensure staining quality, reliability and reproducibility. Conventional fluoresecent miscroscopes and scanners can accommodate image acquisition of the stained slides. This approach offers great flexibility for biomarker research and possibility for IVD product development. Toward the clinical application, a fluorescent mIHC basedIVD test requires the entire workflow including automated staining, whole-slide imaging and image analysis with validated scoring alogorothim.

\section{DISCLOSURE/CONFLICT OF INTEREST}

All authors are full-time employees of Ventana Medical Systems, a member of Roche Group.

1. Dunstan RW, Wharton Jr KA, Quigley C, et al. The use of immunohistochemistry for biomarker assessment-can it compete with other technologies? Toxicol Pathol 2011;39:988-1002.

2. Rimm DL. Next-gen immunohistochemistry. Nature Methods 2014;11: 381-383.

3. Ferri GL, Gaudio RM, Castello IF, et al. Quadruple immunofluorescence: a direct visualization method. Journal of Histochemistry \& Cytochemistry 1997:45:155-158.

4. Lan HY, Mu W, Nikolic-Paterson DJ, et al. A novel, simple, reliable, and sensitive method for multiple immunoenzyme staining - use of microwave-oven heating to block antibody cross-reactivity and retrieve antigens. J Histochem Cytochem 1995;43:97-102.

5. Tornehave D, Hougaard DM, Larsson L-I. Microwaving for double indirect immunofluorescence with primary antibodies from the same species and for staining of mouse tissues with mouse monoclonal antibodies. Histochem Cell Biol 2000;113:19-23.

6. Bauer M, Schilling N, Spanel-Borowski K. Limitation of microwave treatment for double immunolabelling with antibodies of the same species and isotype. Histochem Cell Biol 2001;116:227-232.

7. Toth Z E, Mezey E. Simultaneous visualization of multiple antigens with tyramide signal amplification using antibodies from the same species. J of Histochem Cytochem 2007;55:545-554.

8. Osman TA, Øijordsbakken G, Costea DE, et al. Successful triple immuno enzymatic method employing primary antibodies from same species and same immunoglobulin subclass. Eur J Histochem 2013;57: $143-150$.

9. Stack EC, Wang C, Roman KA, et al. Multiplexed immunohistochemistry, imaging, and quantitation: a review, with an assessment of tyramide signal amplification, multispectral imaging and multiplex analysis. Methods 2014;70:46-58.

10. Pryzwansky KB. Applications of double-label immunofluorescence. In: Bullock GR, Petrusz P (eds). Techniques in Immunocytochemistry, Vol. 1. Academic Press: London, 1982, pp 70-90.

11. Boorsma DM. Direct immunoenzyme double staining applicable for monoclonal-antibodies. Histochemistry 1984;80:103-106.

12. Van der Loos CM, Das PK, Van den Oord JJ, et al. Multiple immunoenzyme staining techniques - use of fluoresceinated, biotinylated and unlabeled monoclonal-antibodies. J Immunol Meth 1989;117:45-52.

13. Pitt JC, Lindemeier J, Habbes HW, et al. Haptenylation of antibodies during affinity purification: a novel and convenient procedure to obtain labeled antibodies for quantification and double labeling. Histochem Cell Biol 1998;110:311-322.

14. Campbell GT, Bhatnagar AS. Simultaneous visualization by light microscopy of two pituitary hormones in a single tissue section using a combination of indirect immunohistochemical methods. J Histochem Cytochem 1976;24:448-452.

15. Nairn RC. Fluorescent Protein Tracing, 4th edn. Churchill Livingstone: Edinburgh, 1976, p 367.

16. Kolodziejczyk E, Baertschi AJ. Multiple immunolabeling in histology: a new method using thermo-inactivation of immunoglobulins. J Histochem Cytochem 1986;34:1725-1729.

17. Wang N, Gibbons CH, Freeman R. Novel immunohistochemical techniques using discrete signal amplification systems for human cutaneous peripheral nerve fiber imaging. J Histochem Cytochem 2011;59:382-390.

18. Feng $Z$, Puri $S$, Moudgil T, et al. Multispectral imaging of formalin-fixed tissue predicts ability to generate tumor-infiltrating lymphocytes from melanoma. J Immunother Cancer 2015;3:47.

19. Pirici D, Mogoanta L, Kumar-Singh S, et al. Antibody elution method for multiple immunohistochemistry on primary antibodies raised in the same species and of the same subtype. J Histochem Cytochem 2009:57:567-575.

20. Gendusa R, Scalia CR, Buscone S, et al. Elution of high-affinity (>10(-9) $\mathrm{K}-\mathrm{D})$ antibodies from tissue sections: clues to the molecular mechanism and use in sequential immunostaining. J Histochem Cytochem 2014;62:519-531.

21. Day WA, Lefever MR, Ochs RL, et al. Covalently deposited dyes: a new chromogen paradigm that facilitates analysis of multiple biomarkers in situ. Lab Invest 2017;97:104-113.

22. Roberts $\mathrm{E}$, Jacob T, Garsha K, et al. Mapping cancer signaling networks by an integrated multiplexed tissue imaging platform. Technology 2016;4:174-193.

23. Garsha K, Ventura F, Pestano G, et al. Multi-modal contrast of tissue anatomy enables correlative biomarker imaging. In: Azar, Intes (eds). Multimodal Biomedical Imaging X. Proceedings of SPIE, Vol. 9316. SPIE: San Francisco, CA, 2015; pp 9316-9317.

24. Buchwalow IB, Minin EA, Boecker W. A multicolor fluorescence immunostaining technique for simultaneous antigen targeting. Acta Histochem 2005;107:143-148.

25. Buchwalow IB, Boecker W. Immunohistochemistry: Basics and Methods. Springer Science \& Business Media: Heidelberg, Berlin, Germany, 2010

26. Suzuki T, Tate $G$, Ikeda $K$, et al. A novel multicolor immunofluorescence method using heat treatment. Acta Medica Okayama 2005;59:145-151.

27. Le Neel T, Moreau A, Laboisse $C$, et al. Comparative evaluation of automated systems in immunohistochemistry. Clin Chim Acta 1998;278:185-192.

28. Moreau $A$, Le Nee $T$, Joubert $M$, et al. Approach to automation in immunohistochemistry. Clin Chim Acta 1998;278:177-184.

29. Prichard JW. Overview of automated immunohistochemistry. Arch of Pathol Lab Med 2014;138:1578-1582.

30. Vermeer AWP, Norde W. The thermal stability of immunoglobulin: unfolding and aggregation of a multi-domain protein. Biophys $J$ 2000;78:394-404.

31. Vlasak J, lonescu R. Fragmentation of monoclonal antibodies. Mabs 2001;3:253-263.

32. Vermeer AWP, Norde W. The influence of the binding of low molecular weight surfactants on the thermal stability and secondary structure of IgG. Colloids Surfaces A Physicochem Eng Aspects 2000;161: 139-150.

33. Gerdesa MJ, Sevinskyb CJ, Sood A, et al. Highly multiplexed single-cell analysis of formalin-fixed, paraffin-embedded cancer tissue. Proc Natl Acad Sci USA 2013;110:11982-11987.

34. Sood A, Miller AM, Brogi E, et al. Multiplexed immunofluorescence delineates proteomic cancer cell states associated with metabolism. JCl Insight 2016;1:e87030.

35. Angelo M, Bendall SC, Finck R, et al. Multiplexed ion beam imaging (MIBI) of human breast tumors. Nat Med 2014;20:436-442. 